\title{
Civilisations
}

Revue internationale d'anthropologie et de sciences

humaines

49 | 2002

Pain, fours et foyers des temps passés

\section{Pains et fours dans le Proche-Orient ancien}

\section{Henri Limet}

URL : http://journals.openedition.org/civilisations/1355

DOI : 10.4000/civilisations. 1355

ISSN : 2032-0442

\section{Éditeur}

Institut de sociologie de l'Université Libre de Bruxelles

\section{Édition imprimée}

Date de publication : 3 juin 2002

Pagination : 37-48

ISBN : 0009-8140

ISSN : 0009-8140

\section{Référence électronique}

Henri Limet, «Pains et fours dans le Proche-Orient ancien », Civilisations [En ligne], 49 | 2002, mis en ligne le 01 juin 2005, consulté le 19 avril 2019. URL : http://journals.openedition.org/civilisations/1355 ; DOI : 10.4000/civilisations.1355

Ce document a été généré automatiquement le 19 avril 2019

(C) Tous droits réservés 


\title{
Pains et fours dans le Proche-Orient ancien
}

\author{
Henri Limet
}

\section{Introduction}

1 La civilisation sumérienne et, à sa suite, assyro-babylonienne était essentiellement agricole et la nourriture de base était le pain. La région se prêtait très bien à la culture de l'orge ( $\beta e$, Hordeum vulgare) à laquelle le climat convenait (son cycle végétatif rapide lui permettait d'arriver à maturité avant les fortes chaleurs) et il supportait la salinité du sol. Dans une certaine mesure, on cultivait aussi l'engrain ( $\mathrm{ziz}_{2}$, Triticum monococcum), beaucoup moins le blé (gig, généralement traduit par «froment ", c'était soit le blé amidonnier Triticum dicoccum, soit peut-être le blé dur, comme de nos jours, Triticum aestivum durum); il faut y ajouter des pois chiches et des lentilles ( $\mathrm{gu}_{2}$ tur-tur et gu ${ }_{2}$ gal-gal, Cicer arietinum et Lens culinaris) auxquels un coin de champ était souvent réservé.

Le signe $\mathrm{NIG}_{2} /$ GAR représente, dans l'écriture, les mots ninda (sumérien) et akalu (accadien) qui ont, à la fois, le sens de " pain » et celui, dérivé, de « nourriture, aliments ». Ceci indique à suffisance combien, en Mésopotamie, se nourrir, c'était avant tout manger du pain ; c'est un fait cultureli. Les courriers qui, à la fin du IIIe millénaire, s'en allaient vers le nord ou vers l'est (voir par ex. B. Lafont, 1985, n 70 à 204), recevaient une ration qui se composait de pain (5 sila par jour, soit environ 4 litres de farine) $)^{\text {ii, }}$ d'huile (de sésame, dans la région et à l'époque), d'oignons et d'un assaisonnement (le naga, produit non clairement identifié), parfois un poisson (à Umma). La ration des corvéables, à la même époque, consistait en céréales en quantités variables selon l'âge, le sexe et, surtout, la compétence du bénéficiaire et de sa place dans la hiérarchie (cette façon de procéder pose d'ailleurs des problèmes aux historiens : que faisaient des surplus non consommés les personnes qui recevaient 15, 20,30, ou même 60 sila d'orge ?). Quant aux documents juridiques, d'une manière générale, quand ils prévoient des allocations aux épouses délaissées ou aux vieux parents, ils mentionnent le $\beta e-b a$, l'i ${ }_{3}-b a$ et le $s i g_{2}-b a$ (ou : $\operatorname{tug}_{2}-b a$ ), 
c'est-à-dire : nourriture en orge, huile et laine (ou vêtement). C'est la base de ce que nous appellerions les besoins élémentaires de l'existence, donc un des aspects de la façon de vivre, en dehors du travail, des relations sociales, des divertissements.

3 Nous apprenons, par l'épopée de Gilgamesh, qu'Enkidu, personnage sauvage qui n'a pas accédé aux pratiques de la civilisation, se nourrit d'herbes comme les bêtes et boit le lait des brebis; quand il est amené à la vie civilisée et urbaine, il regarde avec méfiance le pain et la bière qu'on lui présente ; la prostituée, qui est son initiatrice, lui conseille de les absorber: "mange le pain, lui dit-elle, c'est ce qu'il faut pour vivre; bois de la bière, c'est l'usage du pays » (J. Bottero, 1992: 224 ; tablette P, première moitié du second millénaire).

\section{Le pain}

Mais qu'était-ce, pour un Sumérien, cette nourriture nommée ninda? On pourrait poser la même question au sujet de la bière. Nous traduisons ce terme par «pain " parce que son constituant principal est la farine; ce " pain » ne devait, cependant, pas ressembler au nôtre, mais plutôt à ces crêpes ou galettes azymes que l'on trouve en Syrie actuellement, fort sèches, mangeables quand elles sont fraîches et qui deviennent cartonneuses après quelques heures. Ce n'est cependant pas une certitude. Les Sumériens connaissaient diverses sortes de pain, comme les Grecs anciens qui mangeaient la mâza, la galette d'orge, et l'artos, sorte de miche faite avec du froment.

En sumérien, comme en accadien, les termes ninda et akalu peuvent être accompagnés de diverses qualifications dont la plupart, malheureusement, laissent les historiens dans la perplexité. Pour rester dans les réalités, nous consulterons, en particulier, les archives économiques datant de la 3e dynastie d'Ur (2100-2000 avant l'ère commune). Ce sont des pièces comptables qui reflètent la réalité et non de listes de mots colligées par les clercs. Selon les nécessités, les textes indiquent:

6 a) le type de farine utilisé : on y repère une dizaine de ces farines : $z i_{3}$-se (lire: dabin), $z i_{3}$ $\operatorname{sigx}\left(\operatorname{sig}_{x}\right.$ est la lecture de KAL; c'est une farine destinée au pain dans lequel on a incorporé de la graisse), $z i_{3}$-gum (destinée à un pain de qualité supérieure : $s a g$ ), $z i_{3}-g u$ (qui n'a rien à voir avec la précédente), $\mathrm{zi}_{3}-\mathrm{Be}_{\mathrm{e}} \mathrm{sa}-\mathrm{a}$ "farine d'orge grillée ", $\mathrm{zi}_{3}$-dub-dub (réservée au culte) ; zi ${ }_{3} b a-b a$. On faisait aussi du pain avec de l'eßa, un blé spécial et, bien entendu, avec de l'engrain $\left(z i z_{2}\right)$ ou du froment. Ces distinctions sont probablement dues au degré de blutage ou au mélange de céréales.

b) la forme : ninda kur ${ }_{4}$-a « épais ». Il pourrait s'agir d'une miche.

c) la qualité : gin « ordinaire », $\operatorname{sig}_{5}$ " excellent ». La différence tenait peut-être à la teneur en graisse (voir le d) ou à la présence de sucre (miel, dattes), d'épices diverses (du cumin par ex.).

d) l'élément introduit : le ninda-i $i_{3}$-dé-a « pain dans lequel de la graisse a été introduite ».

e) la façon de le cuire: ninda tum-ru (accad. akal tumri) «cuit sous la cendre »; ina penti «cuit sur des braises».

11 On trouvera une grande variété de mots accadiens désignant les «pains » dans le CAD A/1, à l'article akalu, p. 244.

12 En ajoutant à la pâte du miel, des épices, des pommes ou des dattes, Sumériens et Babyloniens fabriquaient des gâteaux: $g u g_{2} / k u k k u$, dont se régalaient les dieux, à en 
croire certains récits mythologiques : $\operatorname{lu}_{2}$-tur-mu $l u_{2}$ ninda-gug ${ }_{2}-k u_{2}-a-r a$ ninda $-g u g_{2}$ sum-muna-an-ze $e_{2}$-en « donnez des gâteaux à mon jeune ami qui mange (volontiers) des gâteaux " (A. Ferrara, 1973, v. 320-321). Ces nourritures solides sont accompagnées de bière excellente non- mêlée d'eau (Ibid., v. 323 ; littér. « de la bière qui a sa force intacte : a2sikil-la). La déesse Inanna, ailleurs, déguste un gâteau au beurre et se contente d'eau fraîche, mais se hâte de boire ensuite de la bière (G. Flügge, 1973, v. 22 et 28 ; elle prend aussi une autre boisson alcoolisée dite kurun). L'accadien connaît aussi des pains ou gâteaux doux : mutqu (de la racine matâqu « être doux ») et des pains dits kamânu (cuits sous la cendre).

Lugalbanda, héros épique sumérien, mange des deux : ninda mu-ud-gi dingir-re-e-ne-ke ${ }_{4}$ et plus bas ninda gi-de $e_{3}-e \beta$-ta-ba lal ${ }_{3} b a-a n-d u_{8}-d u_{8} l a l_{3} b a-a n-d a-d a h$ "le pain doux des dieux » et « dans le gâteau du miel a été pétri, du miel a été ajouté » (Cl. Wilcke, 1969, v. 51 et 53). Le terme $m u-u d-g i$ (comme tum-ru plus haut) a été emprunté par le sumérien à l'accadien et gi-de ${ }_{3}-e ß$-ta est l'équivalent de kamânu. Dans l'Epopée d'Erra, I,57 (début du Ier millén.), il est dit avec quelque nostalgie: akal ali lullû ul ubbala kamân tumri « le meilleur pain des villes ne vaut pas la galette sous la cendre " (celle que les soldats trouvent dans les camps ; traduction de J. Bottero, $1989: 684)^{\mathrm{iii}}$

Une autre façon de consommer les céréales était encore de les concasser et d'en faire une sorte de gruau, le $n i_{3}-a{ }_{3}-r a$, qui peut-être ressemblait au bourghoul actuel.

A l'opposé, il existait, mais à Mari sur le Moyen- Euphrate, c'est-à-dire à l'ouest, du pain " aigre » (emsu: comparer: geßtin bil ${ }_{2}-l_{2}, k a \beta-b i l_{2}-l a_{2}$ "vin / bière aigre »); peut-être convient-il de traduire par « amer »? J. Bottéro a jadis suggéré de comprendre qu'il s'agit d'un pain fait avec une pâte levée; cette hypothèse a peu de chance d'être retenue (J. Bottéro, 1957 : 257- 258).

\section{La cuisson}

Examinons à présent le problème de la cuisson auquel il a déjà été fait allusion et considérons la phrase suivante extraite d'une incantation : 1 qa ße-am GAL-a (rabâ) GURUÍTUR i-AR-AR-en (itên) ina muhhi SIG4 (libitti) ina IZI(ißati) giß U2-GIR2 (aßagi kamâna inaddi ( CT 39, pl. 24, 30 ; cf. CAD L, p. 178a) : « le jeune homme moudra 1 gros qa ( \pm 1 litre) d'orge et jettera le pain sur le dessus d'une brique dans un feu d'épineux ».

fait, le soin de moudre les grains de céréales (et, éventuellement, de pois chiches) incombait normalement aux femmes : les geme ${ }_{2}$ kikken. Le groupe de servantes auxquelles cette tâche était confiée, était parfois très fourni : 217, par exemple, chacune devant moudre 1 sila $_{3}$ ninda (B. Lafont,1985, $\mathrm{n}^{\circ}$ 256). La meule (na ${ }_{4}$-HAR) était double: sur la pierre inférieure (dite en accadien ßapiltu), très dure, du basalte par exemple, le grain était déposé et il était écrasé par la pierre supérieure (dite elitu) qui tournait sur la première, mue, à la main, à l'aide d'un manche fiché dans un trou ( $\left.n a_{4}-\mathrm{HAR}-\beta \mathrm{u}-\mathrm{si}-\mathrm{ga}\right)$. Il y avait aussi des meules "sans manche». Le travail de moudre le grain est resté du domaine féminin, mais dans un autre contexte, à l'époque suivante: les jeunes filles recevaient en dot, entre autres objets et vêtements, une meule, qui figure aussi dans les inventaires après décès (H. Limet, 1993 : 210 et notes 63- 65).

Nous venons de voir que le feu était alimenté par des épineux. Au temps de la 3e dynastie d'Ur, le gi-izi « roseau à brûler » est souvent cité en grandes quantités (par ex. 3060 bottes, B. Lafont, 1985, n $19 ; 667$ bottes par fagots de 17, dans T. Gomi et S. Sato, 1990, n 474 ; 
660 , ib., $\left.\mathrm{n}^{\circ} 279\right)$; il est glosé en accadien par qanî ßuruptum ou par $\operatorname{Sippatu(CAD} \Omega$, s.v. Sippatu D : 203b et CAD S/3, s.v. ßuruptu)). A. Mari, au XVIIIe s., nous savons qu'on brûlait du bois pour faire du pain : ßurpum, ßuruptum (CAD S/3:373a), mais la ville est située plus au nord et plus à l'ouest. Dans le four à verre, dont il est question dans des textes techniques, il est précisé qu'il fallait des bûches sans noeuds, pelées et assez épaisses, tirées de bouleaux abattus en juillet- août, mais un arrière- plan magique n'est pas exclu qui aurait exigé des manipulations un peu étranges (A.L. Oppenheim, $1970: 32$, tabl.A/B, lignes 16-17).

19 La cuisson à laquelle il est fait allusion plus haut est la plus simple. Le jeune homme devait pétrir une pâte ( $k i r \Omega u$, ce mot désigne, à la fois, un bloc d'argile et une boule de pâte; l'idée commune est la malléabilité). Un texte tardif (cité par CAD E, s.v. epû) conseille: "fais la boule de pâte fais petite, mais le pain large ", c'est-à-dire peu épais (selon la version accadienne). Il s'agit donc d'une abaisse de pâte souple, comme celle que les femmes syriennes, dans les villages, obtiennent avec habileté, ou, du moins, obtenaient, avant la panification industrielle ${ }^{\text {iv }}$. Dans la phrase citée plus haut, le jeune homme devait jeter cette crêpe sur une brique plate, chauffée fortement par le feu de brindilles.

\section{Les fours}

L'installation dont il vient d'être question s'appelait communément, en sumérien, ki.izi, en accadien: kinûnu, ce que les archéologues qui utilisent l'anglais, nomment «fire place », décalque parfait du sumérien : KI «endroit» et IZI «feu». C'est un simple brasier, que l'on pouvait déplacer comme nous en informent les listes lexicographiques : ki-izi-du-du = kinûnu muttalliku, soit: "mobile", c'est ce qu'on nomme des foyers en cuvette (M. Molist, 1985 : 38-39); il faut renoncer à des significations telles que : brasero ou réchaud. Ce dispositif impliquait aussi, comme pour les tannours, des soufflets et l'on tirait de l'un et des autres des présages (E. Reiner, 1958, tabl. II, 109-111)v. La cuisson est directe, si l'on rôtit des brochettes, et elle l'est presque, si l'on interpose une brique ou une plaque de métal.

21 La cuisson «à l'étouffée » est envisageable, mais on imaginera plutôt la fosse-foyer (M. Molist, $1985: 39$ et M. Molist, 1989 : 303 et sv.) ; celle- ci contenait des pierres qui étaient chauffées par des branchages ou des roseaux, le tout recouvert soigneusement, par ex., d'une couche de terre. Ce sont ces types de feu que retrouvent facilement les archéologues ; ils en décèlent la présence à cause des cendrées dispersées aux alentours. Plus élaborés sont les foyers entourés de pierres disposées en cercle, en fer à cheval ou en rectangle, les pierres sont parfois remplacées par une petite levée d'argile.

Les ethnologues connaissent bien ces cavités dans le sol, au milieu de la pièce. On y fait brûler de la bouse de vache séchée, quelques brindilles. Au mieux, la fumée s'échappe par un trou dans le toit; au pis, elle se répand à l'intérieur du local, en faisant tousser et larmoyer les personnes présentes; on en tire d'épaisses rondelles de pain bis, brûléees par endroit et maculées de cendres (W. Thesiger, $1983: 51,59,94)$. En Anatolie, ce foyer s'appelle tandir; Mahmout Makal, dans son ouvrage Bizim köy (M. Makal, 1963: 23 et fig. 3), en donne une description pittoresque, accompagnée d'un dessin ${ }^{\text {vi }}$. Pour éviter les désagréments, les foyers en cuvette et les fosses-foyers étaient rejetés hors des maisons, dans un voisinage plus ou moins proche (ex. en Syrie dans J. Weulersse, 1946: 235- 6, fig. 44 et 45 ; M. Molist, $1985:$ 39). 
Cuire » du pain se dit en sumérien $d u_{8}-d u_{8}$ et, en accadien, epû , qui est parfois précisé ina tinûri. Un proverbe sumérien (E. Gordon, 1959, coll. 2, 29) énonce que le moulin doit être près du four : é na $a_{4}-k i k k e n-n a ~ g u_{2}(i m) \beta u-r i n-n a-k a$. Le terme sumérien, à lire durun ou dilina (M. Civil, 1973 : 172- 5), figure déjà dans l'épopée d'Enmerkar, v. 539 et 542, mais le contexte reste obscur (S.N. Kramer, 1952). Le terme tinûru évoque évidemment le tannûr arabe qui a une forme caractéristique (J. Weulersse, $1946: 231$ et pl. IX ; J.- Cl. Margueron, 199, I : 155) : plus ou moins cylindrique, avec une ouverture au sommet (et non sur le côté) ; les femmes appliquent, au moyen d'un coussinet, la pâte sur la paroi intérieure du tannûr et la récupèrent après quelques instants de cuisson. En consultant les listes lexicographiques (H.-h. X, 339-342), on relève les détails suivants:

- le classificateur IM rangeait le four en question dans la catégorie des objets en argile ;

- le four avait un couvercle : katam tinûri, terme que le sumérien a emprunté, ce qui laisse penser que ce type de four a été introduit par des groupes sémitiques (des nomades?) en Mésopotamie ;

- enfin, le four présentait un bur ${ }_{3} /$ pikallulum « trou, cavité » dont le sens exact n'est pas clair dans le cas présent : est-ce, supposition la plus probable, l'ouverture du sommet, ou peutêtre un trou d'aération à la base?

A. Mari, A. Parrot a retrouvé le fond d'un four voûté avec ouverture à la base (A. Parrot, 1974, fig. 81 et $82 ; \mathrm{J}$.- Cl. Margueron, $1991: 156)^{\text {vii }}$ ainsi que plusieurs fours accolés ; ils se trouvaient dans une cour. Dans d'autres fouilles, il arrive qu'on découvre des amorces de four de ce genreviii ; parfois, les vestiges sont difficiles à identifier et à interpréter. Sur un bas-relief assyrien (de Ninive), souvent reproduit, figure une scène dans laquelle on voit un valet d'armée plonger la main dans une sorte de cuve cylindrique qui pourrait, dit-on, être un tannûr. Plus intéressant est un autre bas-relief (de Nimrud) qui représente diverses activités d'un camp : d'une part, on montre la cuisson sur des braises que ventile un domestique et, d'autre part, la cuisson de pains (?) dans un four (J.Bottero, 1994 : 92-93 et 88).

Le mot tinûru correspond aussi à un autre mot sumérien : udun (passé en accadien sous la forme utûnu ; en arabe : 'attûnu). Le terme utûnu est précisé : c'est le four du potier (glosé également atug pahari ; cf. H.-h. X, 356-362) ; four à griller l'orge : utûn laptix: l'accadien tinûru s'applique au sumérien udun muhaldim «le four du cuisinier », différent donc du kinûnu mais identique au four de potier .

Parmi les signes archaïques d'Uruk, qui sont encore picto-graphiques (derniers siècles du IIIe millénaire), on repère un signe qui évoque très nettement ce type de four; il sert de base à une dizaine de signes complexes : à l'intérieur du dessin principal figure un second, plus petit, qui représente, un poisson, un mouton, une chèvre, le sexe féminin, etc. Ces signes ont disparu; contrairement à d'autres, ils n'ont pas de correspondant dans l'écriture en usage après cette époque. On a proposé d'y voir un chien et d'interpréter les signes complexes comme indiquant des espèces de chiens. Cette hypothèse semble bizarre ; en effet, le mot " chien ", dans ces documents, a son graphème propre (UR) et les dessins dont il est question, n'évoquent nullement cet animal, mais, au contraire, ont un rapport manifeste avec les fours (A. Falkenstein, 1936, n 651 à 661; M.W. Green H.J. Nissen, $n^{\circ} 341$ et sv.).

Dans l'écriture postérieure, le signe UDUN (composé de U + MU) n'a pas de précurseur ; on observe que les clercs ont introduit le signe MU, qui est une allusion au cuisinierboulanger $(\mathrm{MU}=$ muhaldim $)$ : celui-ci y faisait cuire, dans des pots, ragoûts et soupes. On 
attendait que les flammes soient calmées; après qu'on avait enlevé les cendres, les aliments absorbaient la chaleur réfléchie par les parois. Nous avons affaire à un autre type de four qui, lui, est horizontal avec ouverture de face. Il présente, dans certains cas, un foyer distinct d'un « étage », constituant la chambre de chauffe où sont placés, soit les poteries, soit les plats soumis à cuisson indirecte (les plats et moules retrouvés à Mari n'auraient pas supporté une cuisson directe) (A. Parrot, 1974, pl.XXV, fig. 2; J.Cl. Margueron, 1991 : 164). Toutefois, les deux « étages » ne sont pas nécessaires. D’autres variantes sont, du reste, attestées : le feu se trouvant, par exemple, sous le four, devant ou à côté. On observera que ce type de four, au contraire des autres foyers, était installé à l'intérieur des habitations.

Les fouilles de Mésopotamie ont, bien entendu, exhumé, des fours. Ils sont souvent très détruits : cela tient à la nature des constructions en briques de terre séchée. Les fours ne résistent guère à l'écroulement de la maison ou à sa démolition; les murs ruinés étaient sommairement enlevés, puis tassés (ce qui explique, d'ailleurs, la formation des tells) et un nouveau bâtiment s'élevait sur le sol ainsi constitué ; les fours étaient, de ce fait, ensevelis en piteux état. Toutefois, comme ils étaient, ici ou là, adossés à des murs ou établis dans des coins, certaines parties ont résisté et les reconstitutions proposées sont plausibles (G. Delcroix - J.L. Huot, 1972 : 35-82 ; plans : $87-95$; typologie : 95 et sv.).

Pendant longtemps, les fouilleurs n'ont guère prêté attention à ces vestiges de la vie quotidienne et n'ont pas décrit leurs trouvailles avec assez de précision: simples allusions, photos peu lisibles, ni plans ni coupes ${ }^{\mathrm{x}}$. Aussi, pour la Mésopotamie, se réfère-ton généralement aux fouilles menées dans la vallée de la Diyala, sur lesquelles on possède des informations détaillées (OIP 43, pl. X-XI, photos : 51, 39-40 ; 52, 41 ; OIP 58 : 41, fig. 36, 49 , fig. 43, 54, fig.47 ...; OIP $53: 130$ et sv., chapitre Oven and Kilns).

Ce four utûnu n'est pas toujours réservé à la cuisson du pain. En effet, dans certains cas, la sole et la paroi interne présente une couleur rouge qui indiquerait une température très élevée, nettement supérieure aux 300 ou 400 degrés requis pour obtenir du pain.

e) Il existait, enfin, un autre mot en sumérien dont on a de bonnes raisons de croire qu'il désignait un four: gir $_{4}$ et gir $_{4}$-mah. Les contextes dans lesquels il est employé sont explicites : « en ce jour, (le roi fit construire) un gir $_{4}$-mah qui fournit la nourriture de Sin et qui apporte le pain à tous les dieux » (UET VIII, $n^{\circ}$ 67, 37 et sv. ; voir E. Sollberger-J.R. Kupper, 1971 : 188). On cite aussi ce passage du Cyl. A de Goudéa (28,5-6) : « (il fit venir) du gir $_{4}$-mah de grands gâteaux et des croissants " $^{\times \mathrm{i}}$. Le mot accadien kiru qui en a été tiré dans la suite, a été réservé aux fours de bitume et de plâtre, qu'utilisaient, entre autres, les calfateurs de bateaux ; de son côté, kirmahhu c'est-à-dire la forme accadisée de gir $_{4}$ mah, n'est pas, ou guère, entrée dans l'usage, bien qu'elle ait été enregistrée dans les listes lexicographiques. La forme du graphème originel, avec une haute cheminée, est caractéristique.

\section{Conclusion}

Dans les pages précédentes, l'exposé est, avant tout, descriptif et vise à montrer de quelles ressources les Sumériens et les Babyloniens disposaient dans le domaine particulier de l'alimentation, dans les siècles autour de l'an 2000 avant l'ère commune. Cette sociéte était sédentaire, bien qu'il y eût des apports réguliers de nomades ; elle était 
essentiellement agricole, c'est-à-dire que la grande masse de la population s'occupait de la culture du sol et de l'élevage.

L'alimentation avait, comme élément principal, les céréales. La viande n'y jouait qu'un rôle limité. Les énormes troupeaux étaient élevés avec méthode, surtout pour leur laine, mais aussi pour le lait et le cuir; les autorités religieuses y faisaient de larges prélèvements, de chevreaux entre autres, à des fins cultuelles (sacrifices, hépatoscopie). Les boeufs étaient employés aux labours.

Sumériens et Babyloniens avaient élaboré une civilisation dont l'alimentation était basée sur les céréales qui donnaient, d'un côté le pain et, de l'autre, la bière fabriquée elle aussi avec de l'orge. Il faut entendre le terme "pain » au sens large : galettes, crêpes, gâteaux, les uns et les autres de diverses formes et enrichis de fruits, de miel, de graisse et de divers condiments.

Pain et bière les distinguaient donc des populations consommatrices de riz ou de celles qui boivent du vin. Ce n'était pas non plus la chasse, activité de prestige, qui les approvisionnait en viande. En revanche, dans la région méridionale de la Mésopotamie, la pêche en eau douce et en bord de mer leur fournissait un complément de nourriture important.

Dans ces conditions, on comprend que les Sumériens et les Babyloniens aient développé une agriculture bien organisée. Ils connaissaient une grande variété de farines qu'ils accomodaient avec, semble-t-il, beaucoup d'imagination. Au point de vue technique, ils utilisaient plusieurs modes de cuisson, du plus élémentaire (brasier à même le sol, enterré ou légèrement surélevé) jusqu'au four polyvalent.

Fig. 1 - Le tandir, d'après le dessin de M. Makal, 1963 : 23. La fosse, dont le fond est tapissé de pierres, et le conduit qui amène l'air de l'extérieur au début de la combustion et qui est ensuite bouché.

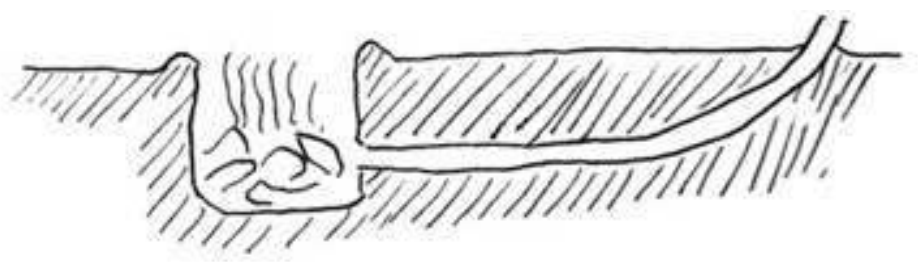


Fig. 2 - Document de Nimrud représentant peut-être un four, d'après J. Bottéro, 1994 :88. Un serviteur ou un cuisinier agite une sorte de éventail au-dessus des vases à la partie supérieure, sans doute pour activer les braises ; des aliments cuisent dans des vases en-dessous, au second étage.

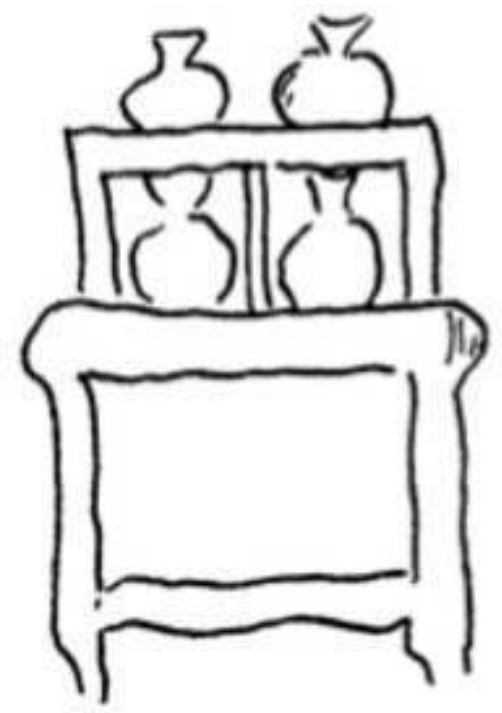

Fig.3 - a) Cuisine 167 du palais Zimri-Lim, à Mari. D'après A. Parrot 1974 : 132, fig.82. Parrot dit avoir retrouvé ces « foyers encore garnis de charbons de bois, prêts à fonctionner ». b) Reconstitution, d'après OIP $53: 130$ et sv. Le feu est allumé et entretenu par l'ouverture située de face et les pots sont disposés sur la sole dans des trous qui assurent leur stabilité et par où passe la chaleur.
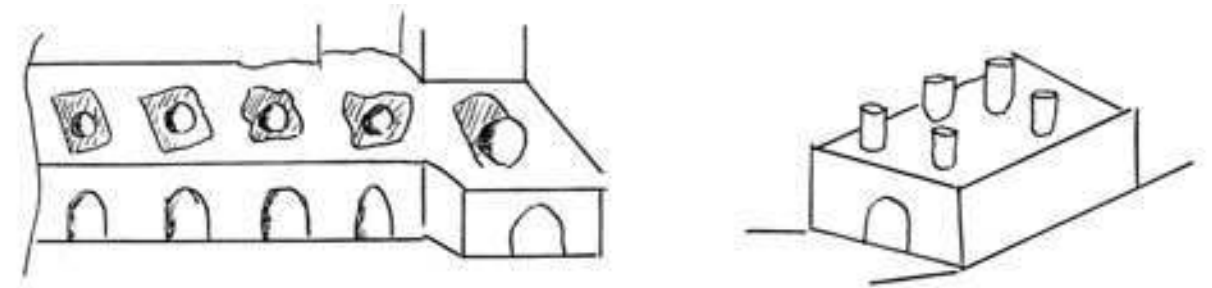

Fig.4 - Pictogrammes qui figurent sur les tablettes archaïques d'Uruk et qui pourraient représenter des fours. D'après A. Falkenstein, 1936
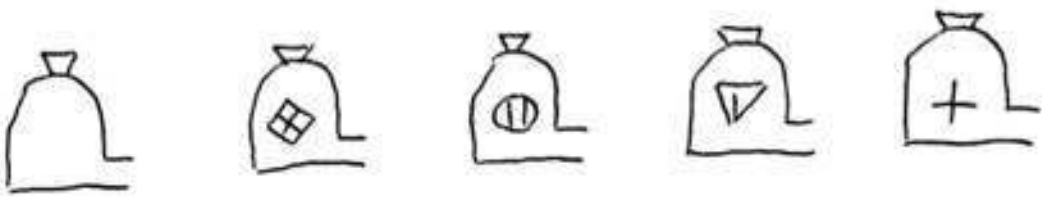
Fig. 5 - Système de four à deux étages séparés par une sole à trous. Au-dessus de la voûte, une ouverture pour l'évacuation des fumées. D'après OIP $53: 130$ et sv.

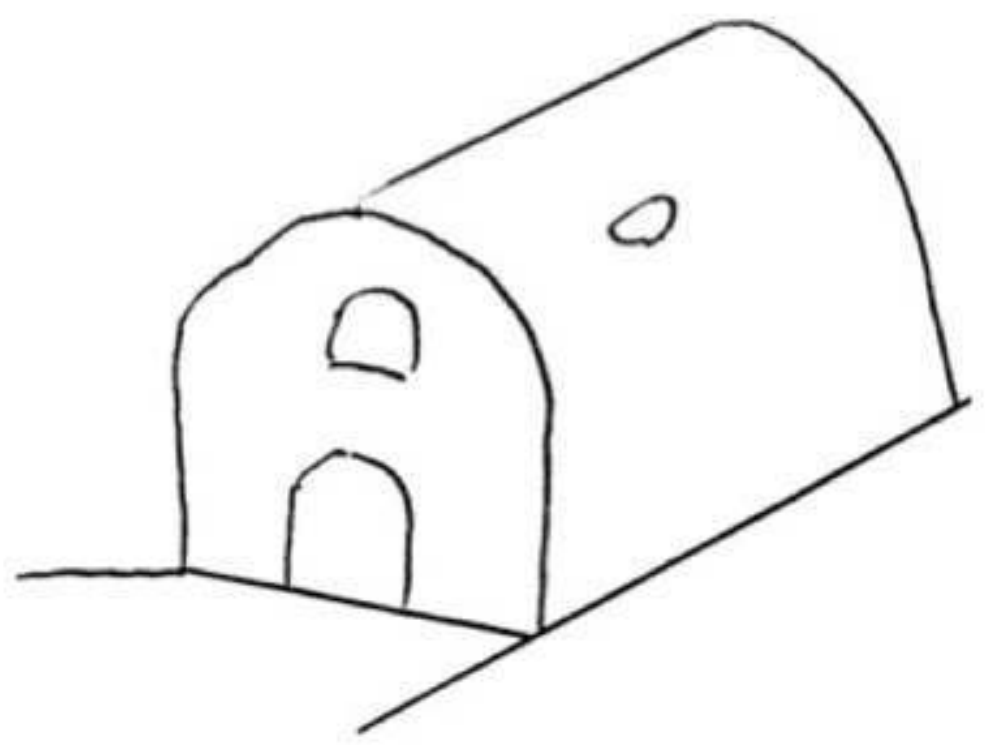

Fig.6 - Schémas de fours, d'après Delcroix-Huot, $1972: 95$, fig.9. a) four simple ; b) four dans lequel la chambre de chauffe et l'alandier sont séparés ; c) four avec foyer enterré. L'entrée d'air est marquée par une flèche.
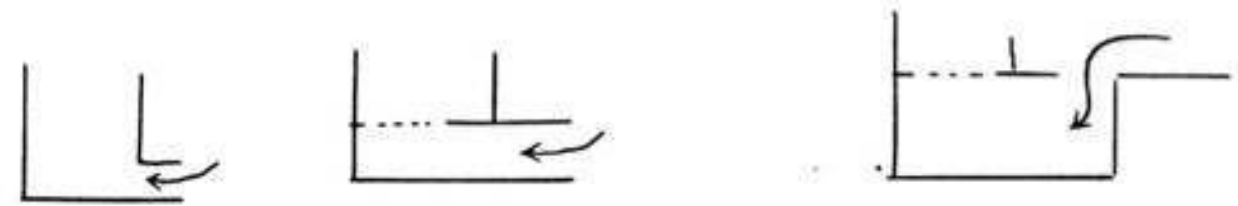

\section{BIBLIOGRAPHIE}

ALSTER B., 1978, « Sumerian Proverb Collection Seven », in Revue d'Assyriologie, 72, p. 97- 112.

BORRERO, J.,1957, Archives Royales de Mari, VII, Imprimerie nationale, Paris.

BORRERO J., 1989, Lorsque les dieux faisaient l'homme, Paris, Gallimard.

BORRERO, J., 1992, L'épopée de Gilgamesh, Gallimard, Paris.

BORRERO, J., 1994, Babylone. A l'aube de notre culture, Gallimard, Paris.

CAD Chicago. Assyrian Dictionary, Augustin Verlag, Glückstadt, 1956.

CIVIL, M., 1973, « Notes on Sumerian Lexicography II », in Journal of Cuneiform Studies, 25.

DELCROIX, G. et HUOT, J.L., 1972, « Les fours dits « de potier » dans l'Orient ancien » in Syria, 49, p. 35-82 et plans, p. 87-95. 
FERRARA, A., 1973, Nanna-Suen's Journey to Nippur (Studia Pohl, ser.maj.2), Inst.Biblique Pont., Rome.

FLÜGGE, G., 1973, Inanna und Enki (Studia Pohl,10), Inst.Biblique Pont., Rome.

T. GOMI - S. SATO, 1990, Selected neo-sumerian administrative texts from the British Museum, ChuoGakuin Univ. (Japon).

GORDON, E., 1959, Sumerian Proverbs. Glimpses of every day life in Ancient Mesopotamia, Museum Monographs, Philadelphia.

H.=h. Série lexicographique HAR-ra=hubullu .

KRAMER, S.N., 1952, Enmerkar and the Lord of Aratta, Philadelphia.

LAFONT, B., 1985, Documents administratifs sumériens, Ed. Recherche sur les Civilisations, Paris.

LIMET, H., 1993, La famille et la vie privée à l'époque paléo-babylonienne, in Íulmu IV, Poznan, p.199-212.

MAKAL, M., 1963, Un village anatolien, trad. française (Coll.Terrre humaine/Poche), Plon, Paris.

MARGUERON, J.-Cl.,1991, Les Mésopotamiens 1, A.Colin, Paris

MEIER, G., 1937, Die assyrische Beschwörungssammlung Maqlû, in Archiv für Orientforschung. Beiheft 2, Berlin.

MOLIST, M., 1985, in Cahiers de l'Euphrate, 4.

MOLIST, M., 1989, Problématique des structures de combustion, in Mémoires du Musée de préhistoire d'Ile de France, 2.

OIP Oriental Institute (Chicago), Publications.

opPENHEIM, A.L., 1970, Glass and Glassmaking in Ancient Mesopotamia, Corning, New York.

PARROT, A., 1974, Mari. Capitale fabuleuse, Payot, Paris.

REINER, E., 1958, «Íurpu. A collection of Sumerian and Akkadian incantations, » in Archiv für Orientforschung. Beiheft 11, Graz.

THESIGER, W., 1983, Les Arabes des marais, trad. française (Coll.Terre humaine/Poche), Plon, Paris.

UET VIII, Ur Excavations. Texts. Ed. E.Sollberger, Londres, 1965.

WEULERSSE, J., 1946, Paysans de Syrie, Gallimard, Paris.

WILCKE, Cl., 1969, Das Lugalbandaepos, Wiesbaden.

\section{NOTES}

i. On remarquera qu'en français, une évolution analogue mais inverse s'est produite avec le mot « viande » qui avait primitivement le sens de nourriture (italien vivanda) et qui a pris celui, plus restreint, d'aiment carné.

ii. L'alimentation des nomades et leur ignorance des usages des sédentaires en la matière sont sujets d'étonnement, comme le montre le proverbe: " un gâteau avec de la farine de pois au lieu de miel, le nomade le mange sans reconnaître ce qu'il y a dedans “ (B. Alster, 1978, nº 95) .

iii. Le CAD A/1, p. 242 a, qui cite ce passage, en mentionne un autre avec l'expression akal pitim "le pain cuit sur les braises “. 
iv. On voyait encore faire la pâte de cette façon en Syrie, il y a quelques années, dans les villages. Les femmes utilisaient cependant le tannour. Sur l'utilisation moderne des foyers en cuvettes, cf. M. Molist, 1985, p. 41 .

v. " Il a demandé un signe par le fourneau embrasé “ (ina KI.IZI nap-hi), puis " par la torche ", enfin " par les soufflets ".

vi. Un conduit souterrain amène l'air de l'extérieur, mais, dès que le feu est bien allumé, on va boucher l'orifice avec un vieux lambeau d'étoffe ; on devine le résultat.

vii. Sur la fig. 82, dans l'ouvrage de Parrot, il s'agirait plutôt de réchauds : on y posait des marmites ou des poeles. Pour les autres figures, ce serait des fours à pain.

viii. Dans OIP 97, pl.18b et commentaire p. 19 : four ovale avec des murs de $18 \mathrm{~cm}$ d'épaisseur et préservés sur $18 \mathrm{~cm}$ de hauteur. Il semble qu'on a affaire à une construction en dôme, en partie en encorbellement (la date : dynastique archaïque III, soit vers 2500 ).

ix. Dans la série d'exorcisme Maqlû, IV, 69 (G. Meier, 1937) sont énumérés les divers types de fours; ils étaient donc bien distingués dans la pratique et le vocabulaire: ana utÛn lapti, tinûri, kinûni .

x. Observation de Delcroix et Huot (1972) dès le début de leur article. Les choses ont heureusement changé depuis quelques années. Les fouilleurs sont plus attentifs aux vestiges de ma vie quotidienne et la bibliographie devient plus étoffée. Cf. les études de M. Molist citées plus haut.

xi. La traduction par " croissant " est de A. Falkenstein, mot à mot " grande corne ", sumér. sigal ; un autre terme est si-gug " gâteau en forme de corne " (A. Ferrara, 1973, v. 325).

\section{RÉSUMÉS}

La civilisation sumérienne était essentiellement agricole et les habitants de l'ancienne Mésopotamie mangaient surtout des cérales : l'orge, le blé. D'après les documents économiques, en particulier ceux de la IIIème dynastie d'Ur, il existait une grande variété de farines de pains. Dans la seconde partie de cet article, les différentes étapes de la préparation du pain sont expliquées. Une attention particulière était accordée à la cuisson du pain. La pâté pouvait être disposée sur des pierres chauffées. Un four appelé tinuru en acadién présentait un système plus compliqué; il était fait d'argile et correspondait, peut-être, à une sorte de tannour, tel qu'il est connu dans le monde arabe moderne. L'utunu était plutôt un four de potier qui pouvait aussi être utilisé par le cuisinie.

The Sumerian civilisation was mainly agricultural and the inhabitants of ancient Mesopotamia were eating avove all cereals : barley, wheat. In the economic documents, particularly those of the IIId dynasty of Ur, many varieties of flour and bread are mentioned. In the second part of this paper, the preparation of bread is explained in its different stages. A special attention is paid to the baking of the breads. The dough could simply be put on hot stones. An oven, named tinuru in akkadian, showed a more complicated system; it was made of clay and, maybe, was a kind of tannûr, as it is known in the modern arabic area. The utunu was rather a kiln that was used by the potter but also by the cook. 
INDEX

Keywords : Bread, ovens, cereals, Mesopotamia, Sumerian civilisation

Mots-clés : Pains, fours, céréales, Mésopotamie, civilisation sumérienne

\section{AUTEUR}

\section{HENRI LIMET}

Histoire et Philologie Orientales, Université de Liège, Belgique. 